\title{
Human Gait-labeling Uncertainty and a Hybrid Model for Gait Segmentation
}

\author{
Jiaen Wu, Henrik Maurenbrecher, Alessandro Schaer, Barna Becsek, Chris Awai Easthope, George \\ Chatzipirpiridis, Olgac Ergeneman, Salvador Pané, Member, IEEE, Bradley J. Nelson, Fellow, IEEE,
}

\begin{abstract}
Motion capture systems are widely accepted as ground-truth for gait analysis and are used for the validation of other gait analysis systems. To date, their reliability and limitations in manual labeling of gait events have not been studied.

Objectives: Evaluate human manual labeling uncertainty and introduce a new hybrid gait analysis model for longterm monitoring.

Methods: Evaluate and estimate inter-labeler inconsistencies by computing the limits-of-agreement; develop a model based on dynamic time warping and convolutional neural network to identify a valid stride and eliminate nonstride data in walking inertial data collected by a wearable device; Gait events are detected within a valid stride region afterwards; This method makes the subsequent data computation more efficient and robust.
\end{abstract}

Results: The limits of inter-labeler agreement for key gait events of heel off, toe off, heel strike, and flat foot are $72 \mathrm{~ms}$, $16 \mathrm{~ms}, 22 \mathrm{~ms}$, and $80 \mathrm{~ms}$, respectively; The hybrid model's classification accuracy for a stride and a non-stride are $95.16 \%$ and $84.48 \%$, respectively; The mean absolute error for detected heel off, toe off, heel strike, and flat foot are $24 \mathrm{~ms}, 5 \mathrm{~ms}, 9 \mathrm{~ms}$, and $13 \mathrm{~ms}$, respectively.

Conclusions: The results show the inherent label uncertainty and the limits of human gait labeling of motion capture data; The proposed hybrid-model's performance is comparable to that of human labelers and it is a valid model to reliably detect strides in human gait data.

Significance: This work establishes the foundation for fully automated human gait analysis systems with performances comparable to human-labelers.

Index Terms_-Digital biomarker, manual gait events, convolutional neural network, dynamic time warping, automatic gait analysis, wearable inertial sensors.

\section{INTRODUCTION}

This work was supported by the European Union's Horizon $2020 \mathrm{Re}$ search and Innovation program under the Marie Sklodowska-Curie grant agreement No.764977. (Corresponding author: Alessandro Schaer)

J. Wu, H. Maurenbrecher, S. Pané, B. J. Nelson are with the Institute of Robotics and Intelligent Systems, ETH Zurich, Tannenstrasse 3, CH-8092 Zurich, Switzerland (e-mail: wujiae@ethz.ch; henrikm@student.ethz.ch; vidalp@ethz.ch; bnelson@ethz.ch).

J. Wu, H. Maurenbrecher, A. Schaer, B. Becsek, G. Chatzipirpiridis, O. Ergeneman are with Magnes AG, Selnaustrasse 5, 8001 Zurich, Switzerland. (e-mail: aschaer@magnes.ch; barna@magnes.ch; chgeorge@magnes.ch; oergeneman@magnes.ch).

C. A. Easthope is with cereneo Foundation, Center for Interdisciplinary Research (CEFIR), Vitznau, Switzerland (e-mail: chris.awai@cereneo.foundation).
$\mathbf{H}$ UMAN walking is a complex dynamic process. It integrates multiple interdependent sensory inputs and requires coordination of the motor outputs to achieve efficient, stable, and adaptive locomotion. The deterioration of human gait can severely affect people's mobility, reduce the activities of daily life, and affect the quality of life. In general, gait can be described by its temporal and spatial characteristics such as stride time, stride length, stride velocity, swing time, stance time, and their corresponding variability and asymmetry metrics [1]. Probing and evaluating these gait characteristics is important not only in the field of rehabilitation [2]-[4], but also in the early detection of neurological diseases, such as Parkinson's disease [5], [6], Alzheimer's disease [7], [8], tremor syndromes [9], [10], and stroke [2], [11]. Some specific gait patterns even appear in the earliest stage of neurological diseases before other signs and symptoms are evident, providing significant clues for early diagnosis and treatment [12][14]. Therefore, quantitative gait characteristics can be used as a digital biomarker for monitoring and assessing people's health status.

To assess and evaluate people's gait by temporal and spatial characteristics quantitatively, each gait cycle is divided into two main phases i.e., swing phase and stance phase [15]. These two main phases can be further divided into eight sub-phases by gait events within one gait cycle: initial contact, loading response, mid-stance, terminal stance, pre-swing, initial swing, mid-swing, and terminal swing [16]. Key gait events segmenting these phases are heel strike (HS) and toe off (TO). Additional events of interest include heel off (HO) and flat foot $(\mathrm{FF})$, which give insights into the transition periods between these two main gait phases. For pathological gait, the proceeding sequence and the time allocation of gait phases can vary compared to healthy subjects, and some gait events or phases might even be missing completely [17], [18]. Observing these variations in gait events during long-term daily activities provides a powerful tool for clinical gait assessment, evaluation, and diagnosis [19], such as identifying early Parkinson's disease (PD) [20], predicting freezing of gait [21], [22], monitoring PD symptoms [23], and the Levodopa response [24]. In light of the ongoing shift of rehabilitation and early diagnosis from physical visits of care facilities toward remote monitoring systems, the understanding of real-world gait data gains significant importance [25]. Recent studies have investigated the relationships between clinical gait assessments and home-based monitoring systems, and have described an 
additive component for real-world data [19], [26]. A reliable method for continuous remote monitoring of gait quantity and quality affords new perspectives of assessing the real-world impact of interventions and of investigating the transfer of rehabilitation progress to out-patient behaviour.

To capture the quality of gait, a significant research effort has been made towards achieving a comprehensive understanding of spatio-temporal gait characteristics, such as motion capture systems [27], [28], pressure mats [29], [30], and wearable inertial sensors [31]-[33]. Compared to traditional subjective visual observations by therapists, instrumented assessments allow for a more objective assessment of gait characteristics [34]. Motion capture systems give a detailed evaluation of motion while requiring a complex stationary set-up involving cameras and the collected data needs to be analyzed manually to identify relevant gait characteristics. This makes these gait systems very expensive to operate, and the reported data complex to interpret. Lastly, motion capture systems cannot track subjects outside the laboratory, and therefore, data collection is limited to discrete assessment time points at which the patient must physically visit the facility. An alternative is pressure mats, which measure ground reaction forces when people are treading on them. While the set-up has reduced complexity, gait measurement is still confined to a gait laboratory, and the available range of gait parameters and information from pressure mats is limited. Both motion capture systems and pressure mats, offer an ideal walking environment, allowing individuals to focus their attention solely on ambulation. This may lead to biased results, as the recorded gait is not representative of the patient's typical gait. Additionally, the measurements often take place under the supervision of a health professional, which may further skew the collected data [35], [36]. These costly and laboratorylimited data acquisition systems have led to a growing urge for cheaper and mobile systems. Combined with the development of micro electro-mechanical systems (MEMS), the use of inertial sensors as wearable devices for gait analysis has gained importance and attractiveness in mobile gait analysis [31] [33]. Wearable inertial sensors are not only cost-effective and portable, but can also be used for a longer period of time of continuous monitoring with unbiased measurement during daily routine.

For validation of wearable inertial sensors, optical motion capture systems are the most commonly used reference systems and are considered as the gold standard [37], [38]. They employ either retro-reflective (passive) or infrared (IR) emitter (active) markers attached to different locations on the lower limbs. These markers are tracked through multiple cameras to record spatial gait trajectories. The key gait events are then labeled by technicians who visually examine the markers' spatial trajectories or by automated event detection algorithms based on markers' position and velocity [39], [40] for further gait parameter estimation. Although these automated detection algorithms are time efficient, they are unviable in patient population due to the high variability that is intrinsic to pathological gait. Manual labeling is still the most widely accepted benchmark for validation of wearable inertial devices for gait analysis [33], [37], [41]. Nonetheless, the uncertainty of manual labels and the limit of detection have never been considered and evaluated. The uncertainty in manual labels mainly comes from two sources: 1) the lack of consistency within a labeler i.e., intra-labeler inconsistency; and 2) the lack of consistency among different labelers i.e., inter-labeler inconsistency. The label quality of gait events and acquisition results are highly dependent on the labeler's personal perspective and experience level. The uncertainty of labels in gait data resulting from intra-labeler and inter-labeler inconsistency can have a direct impact on the reliability of gait event labels, and further affect the accuracy of gait parameter estimation and the decision by doctors. Therefore, one purpose of this study is to investigate the uncertainty in manual labeling in the well-accepted benchmark i.e., optical motion capture systems.

Moreover, to estimate spatial and temporal gait parameters from inertial wearable sensors, different algorithms have been developed. The first group of gait analysis methods relies on straight gait event detection in sensor signals [42]. Gait events can be identified by different techniques, including rulebased [43], [44] and machine learning methods [45], [46]. The second group of methods split gait analysis into two stages: first segmenting the sensor data into strides, and then searching for gait events within each found stride. The advantages of identifying single strides first are that it is more robust for pathological gait where some gait events may be missing [42], and more efficient for long-term gait recording processing since it rejects non-stride data in the first stage, reducing the computational cost [47]. This two-stage gait analysis has been investigated in the literature with many techniques, such as thresholding/peak detection methods [47], [48], template matching methods [49], [50] and machine learning-based methods [51].

Ullrich et al. extracted "free-living" (uncontrolled) strides by detecting the peak of harmonic frequency in the frequency domain of accelerometer and gyroscope signals measured from the foot [47]. The authors reported a high sensitivity of $98 \%$ and specificity of $96 \%$ for stride detection. Hickey et al. identified and quantified strides and bouts of healthy subjects by thresholding an accelerometer data measured on the lower back [48]. The authors showed a high sensitivity of gait detection and false positives for non-gait classification by mistaking intense cycling for walking.

Oudre et al. detected walking strides of both healthy and pathological subjects [49]. The authors applied a template matching approach on accelerometer and gyroscope signals measured at the instep. In this approach, the recall and precision reached up to $98 \%$ and $98 \%$. Barth et al. isolated free walking strides from shoe-mounted gyroscope and accelerometers by searching a pre-defined stride template with multi-dimensional subsequence dynamic time warping (DTW) approach [50]. The authors achieved a high precision of $97 \%$ and recall of $97 \%$. While with those template matching only methods, an empirical defined rule is still necessary to define the probability of being a stride. Robust algorithms for automatic stride detection and non-stride rejection from longterm continuous gait monitoring are crucial for efficient gait analysis. Therefore, in this paper, a hybrid stride segmenting 
approach based on DTW and convolutional neural network (CNN) is proposed to identify stride signals and eliminate nonstride signals from inertial sensor data.

The contribution of this study is two-fold: 1) A method has been provided for estimating and analyzing the uncertainty in manual labeling of human walking data from an optical motion capture system. To measure the labeling uncertainty, five labelers were recruited and given the task to manually label the key gait events (HO, TO, HS, and FF) on markers' positional trajectories from the camera-based motion capture system. A total of almost 28000 labeled events were obtained and further analyzed. The uncertainty and the limit of agreement (LOA) for gait event detection accuracy are estimated and analyzed. For four key gait events of HO, TO, HS, and FF, LOA are $72 \mathrm{~ms}, 16 \mathrm{~ms}, 22 \mathrm{~ms}$, and $80 \mathrm{~ms}$ respectively. 2) An assembling gait cycle identification approach is developed. An automatic inertial-sensor-based stride detection algorithm based on DTW and CNN named stepperNet is first introduced to automatically and reliably identify gait strides and reject non-strides from walking signals. A gait event detection algorithm based on identifying specific signal characteristics is later employed to segment the stride into sub-phases for further analysis. This method allows for intra-stride-based event detection rather than searching gait events through the entire walking recording, which makes the algorithms more efficient. It also shows potential for adapting this approach towards pathological gait and achieving personalized gait analysis.

\section{Data Collection}

\section{A. Subjects of the Study}

Four healthy subjects were recruited to participate in this study. They were labeled as subject $P_{1}-P_{4}$. All subjects were able to walk normally and had no known injuries or abnormalities that affected their gait. Written informed consent was provided by all participants and this study was conducted in accordance with Good Clinical Practice guidelines and the Declaration of Helsinki after receiving a declaration of clearance from the local ethics committee (BASEC Nr Req2019-00715).

\section{B. Wearable Gait Analysis System}

Gait was monitored with the wearable gait analysis system (WGAS) shown in Fig. 11 It consists of a pair of shoes with custom-developed embedded electronics. The electronics include a wireless-enabled (BLE and $\mathrm{WiFi}$ ), dual-core MCU (ESP32, Espressif Systems CO Ltd, Shanghai, China) running a custom FreeRTOS-based ${ }^{1} \mathrm{C}$ firmware for handling communication with a custom iOS application, and on-board sensors (LSM6DSM and LSM303AGR, STMicroelectronics, Geneva, Switzerland). The system allows logging sensor data to a local SD card or wirelessly streaming to a mobile device through BLE. WGAS also includes vibration-motors for haptic-feedback and/or general signaling to the user, which was utilized for the synchronization of different systems in this study.

\footnotetext{
${ }^{1}$ https: / / www. freertos.org
}

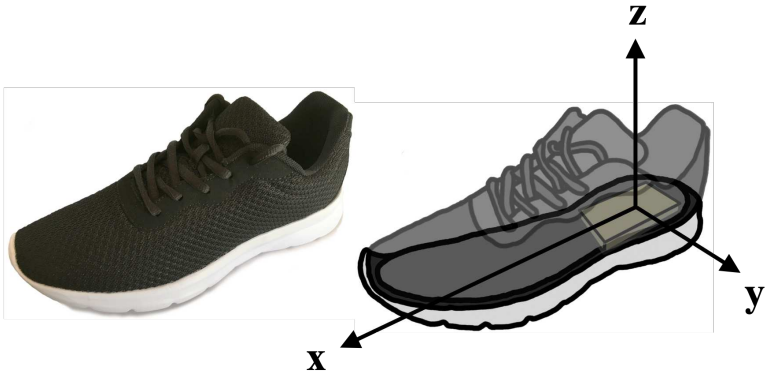

Fig. 1: Schematic illustration of WGAS ecosystem embedding sensors in the outsole of the shoes.

The shoes can be paired with an application running on a smart device, e.g. a smartphone, through which the user can adjust settings and retrieve data from the shoe. According to the needs of this research, the range of accelerometer, gyroscope, and magnetometer are set at $\pm 8 \mathrm{~g}, \pm 2000^{\circ} / \mathrm{s}$, and 50 gauss, respectively. The sampling frequency of data acquisition is set at $100 \mathrm{~Hz}$. The data acquisition mode is set to logging data to the on-board SD card. The sensor's body frame, in which the acceleration, angular rate, and magnetic field are measured, can be seen in Fig. 1. The $x$-axis is aligned with the shoe's major principal axis from heel to toe, while the $z$-axis is normal to the shoe's sole and pointing upward. Lastly, the $y$-axis is set in accordance to $x$ and $z$, such that the frame is right-handed.

\section{Experimental Setup and Data Acquisition Protocol}

Subjects were instructed to wear the sensor-embedded WGAS shoes and perform consecutive walking with a normal gait on a treadmill. They were guided to complete three trials without support at $N_{v}=3$ different walking speeds spanning typical locomotion speeds for early community ambulators to healthy walkers, specifically $0.53 \mathrm{~m} / \mathrm{s}, 0.86 \mathrm{~m} / \mathrm{s}$, and $1.11 \mathrm{~m} / \mathrm{s}$. The treadmill (Bertec) was equipped with two force plates beneath the treadmill belt measuring ground reaction force sampled at $600 \mathrm{~Hz}$. Retroreflective markers $(14 \mathrm{~mm})$ were placed on subjects' shoes at the first and fifth metacarpal joints, the tip of the second distal phalanx, and at the end of the calcaneal tuberosity, as also on the medial and lateral malleolus of the ankle. Positional data of markers were collected by the motion capture system (Qualisys Opus 400 with 9 cameras) running at $100 \mathrm{~Hz}$.

The data-acquisition protocol involved the following stages:

- A simple calibration process was carried out before each walking trial began, consisting of turning-on and -off of the vibration on both shoes to give a signal for data synchronization. The vibration was registered by WGAS as a binary signal (ON/OFF) and by the treadmill's force-plates. The registered vibration signals allowed for synchronization of the motion capture data and WGAS data in post-processing.

- WGAS immediately started logging thereupon.

- The treadmill was subsequently ramped up to the respective speed and the subjects were asked to acquaint 
themselves with the speed.

- Once the treadmill was at final speed and the subjects were comfortable walking at its speed, they performed a pronounced clapping motion with their hands to signal the start of the steady-state motion capture measurement.

- Subjects then walked for approximately $N_{s}=50$ strides for left and right foot, respectively. Stride count was monitored by a dedicated person.

- After completing 50 strides on each side, the motion capture measurement was terminated, followed by a stop of WGAS logging and a halting of the treadmill.

This protocol was repeated for each subject and speed. Data obtained from the motion capture system contained the markers' positional data in three orthogonal axes denoted as $\vec{p}=\left(p_{x}, p_{y}, p_{z}\right)$, and the magnitude of the exerted force on the treadmill including its orientation vector in three axes, denoted as $\vec{F}=\left(F, n_{1}, n_{2}, n_{3}\right)$. Data obtained by WGAS consisted of acceleration, angular velocity and magnetic field vectors in three axes, which can be denoted as $\vec{a}=\left(a_{x}, a_{y}, a_{z}\right)$, $\vec{\omega}=\left(\omega_{x}, \omega_{y}, \omega_{z}\right)$, and $\vec{H}=\left(H_{x}, H_{y}, H_{z}\right)$, respectively.

\section{HUMAN LABELING}

\section{A. Labeling Strategy}

We assess the uncertainty in human gait labeling, which is widely accepted as the gold standard. The same datasets were labeled by $N=5$ labelers. We analyze the variation of labels bases on different labelers. The labelers were trained to use Mokka 3D Motion Kinematic \& Kinetic Analyzer version 0.6.2 [52] to identify the key gait events, i.e., HO, TO, HS, and FF for both left and right feet. Note that different gait terminologies are used in different works [53]. In this paper, we consider the wording of $\mathrm{HO}, \mathrm{TO}, \mathrm{HS}$, and $\mathrm{FF}$ as four gait events. Within a stride, the following definitions for the events apply: $\mathrm{HO}$ is defined as the first sample at which the heel (marker) leaves the ground after a FF event; TO is defined as the first sample at which the toe (marker) leaves the ground after a HO event; HS is defined as the first sample at which consistent contact between the heel and the ground is achieved after the swing phase; and FF is defined as the first sample at which the tip of the foot is flat on the ground after an HS event. All labelers were given the same task of labeling three datasets $(1.11 \mathrm{~m} / \mathrm{s}, 0.86 \mathrm{~m} / \mathrm{s}, 0.53 \mathrm{~m} / \mathrm{s})$ for each of the $N_{h}=$ 4 validation subjects $P_{1}-P_{4}$. This corresponds to roughly $N_{L}=2 \cdot N_{s} \cdot N_{v} \cdot N_{h} \cdot N_{e}=4800$ events manually labeled by each labeler, leading to $N_{E}=N \cdot N_{L}=24000$ labeled events obtained in total.

\section{B. Labeling Caveats}

In general, labeling is not a difficult task for a human being, while it comes with some caveats, such as lossy marker data due to marker occlusion, the lack of consistency within a labeler, and the lack of consistency among different labelers.

Labeling uncertainty is thus to be expected, and introduces a new problem: one cannot simply compute statistics on the

\footnotetext{
2 https://github.com/Biomechanical-ToolKit/Mokka
}

manual events by constructing a matrix with the labels' arrays by all labelers since

1) We have no a priori guarantee that all labelers labeled the same number of labels;

2) Even if all arrays have the same length, it does not mean that a 1:1 correspondence is at hand.

To illustrate these issues, the reader is referred to Fig. 2a in the depicted example, the two sequences $X$ and $Y$ from two labelers contain the same number of events, while assuming a 1:1 correspondence would lead to an artificial error. Two of the labels are to be considered as outliers and an algorithm capable of matching events for accurate error computation is needed.

\section{Labeling Condensation Process}

1) Solution Description: Given the uncertainty in the manual labels, we need to be able to match each label of labeler $X$ to the corresponding label of labeler $Y$. This is necessary for each pair of labelers $\{X, Y\}$ for $X, Y \in\{Z: Z$ labeler $\}$. To achieve this labels-condensation, we apply a matching algorithm iteratively among pairs of labelers until the number of labels for each labeler converges to the same number. This is done for each gait event, side (left or right), and experiment (walking speed and walking subject) individually.

The matching algorithm we use herein is our implementation of the Needleman-Wunsch algorithm (NWA) used in DNA sequences matching [54]. The basic idea is to apply dynamic programming to find the optimal alignment of two sequences while allowing for the insertion/deletion of entries from either sequence. We implement the NWA in Python3 with numpy. We define the costs as follows:

- Match cost $e_{m}=r_{i}-x_{j}$ with $r_{i}$ being the reference sequence value at index $i$ and $x_{j}$ being the test sequence value at index $j$;

- Insertion/deletion cost $e_{d}=e_{i}=\mathrm{E}[\Delta(\vec{r})]$, where $\mathrm{E}[\cdot]$ denotes the expected value operator, $\Delta(\cdot)$ is the firstorder, forward finite difference operator, and $\vec{r}$ is the array of reference values.

We loop over all labelers starting with an arbitrary labeler as the reference labeler and apply the matching algorithm. Once all labelers have been compared to the reference labeler, we check whether all labelers agree on the number of labels for the specific event, side, speed, and subject. If an agreement is reached, i.e. all labelers are left with the same number of labels, we proceed with the next case, else we change the reference labeler and repeat the matching. This process is repeated until convergence is reached. This algorithm is illustrated in Algorithm 1 and a depiction of its effect can be found in Fig. $2 b$.

\section{Matching Results}

In all cases, our implementation of the NWA showed satisfactory results meaning a very small number of labels were deleted from the data while still ensuring usable results and a minimum number of iterations. The maximum number of deleted events was 23 out of 333 available (for $P_{4}, 1.11 \mathrm{~m} / \mathrm{s}$, 


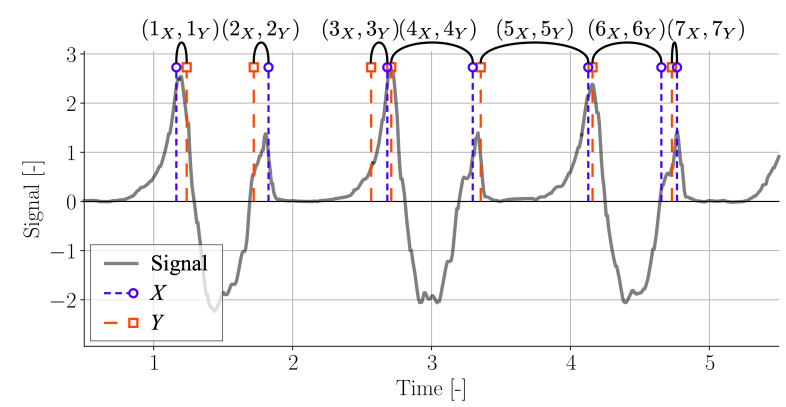

(a) Naive matching: events are matched according to their index; excessive events are discarded (not depicted). This approach introduces bigger errors than what is to be expected in the final error computation. In this example, matching errors occur for the identified pairs $\left(4_{X}, 4_{Y}\right),\left(5_{X}, 5_{Y}\right)$, and $\left(6_{X}, 6_{Y}\right)$.

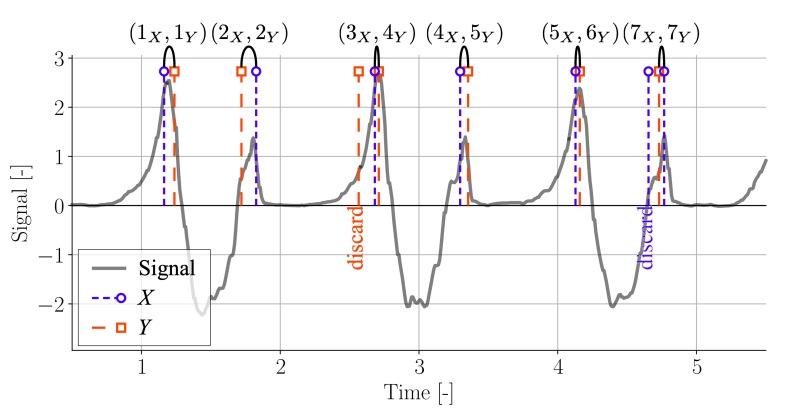

(b) Optimal matching: events are matched according to their relative distances and some events are discarded, namely $Y[3]$ and $X[6]$ - these are completely discarded in the error computation as they are considered as outliers. The reasons for the existence of such outliers are multiple and they include human errors as well as automatic labeling errors.

Fig. 2: Events-matching problem illustration: given a signal and two (individually ordered) sequences of events $X$ and $Y$ related to the signal, it is demanded to find the best sequence of index pairs $\left(i_{X}, i_{Y}\right)$, associating elements of $X$ to elements of $Y$ such that one can then proceed in computing the error between $X$ and $Y$. The two illustrations above show: a) a naive association process, where labels with the same index are paired; b) the desired, optimal solution to the problem, where, in this very specific case, one entry of $X$ and one entry of $Y$ are dropped. A similar problem was found and addressed in the analysis of DNA sequences by Needleman and Wunsch [54] and their algorithm is used in this work to match labels from different human labelers and to match events from human labeling and algorithmic labeling.

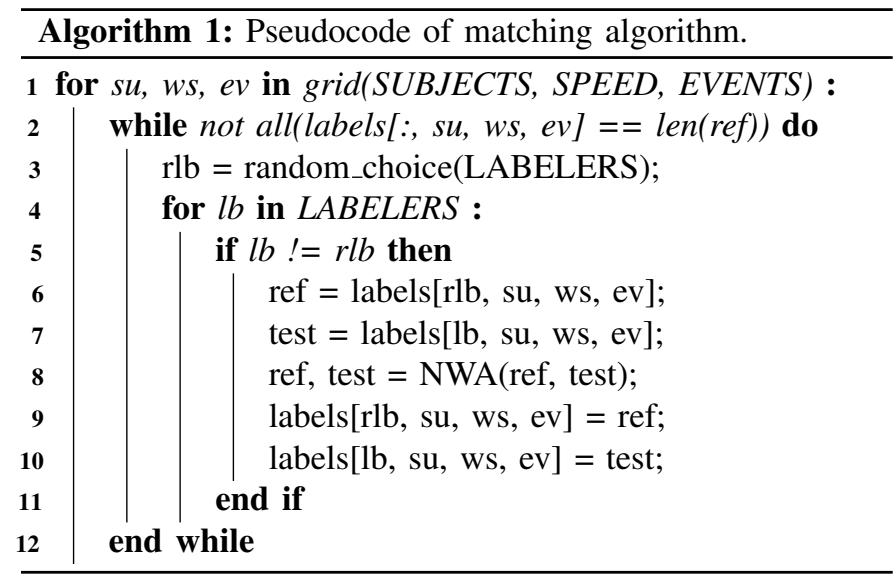

\begin{tabular}{|c|c|c|c|}
\hline Event & Labels & Removed & Fraction \\
\hline HO & 6916 & 121 & $1.75 \%$ \\
\hline TO & 6930 & 95 & $1.37 \%$ \\
\hline HS & 6924 & 84 & $1.21 \%$ \\
\hline FF & 6913 & 113 & $1.63 \%$ \\
\hline Total & $\mathbf{2 7 6 8 3}$ & $\mathbf{4 1 3}$ & $\mathbf{1 . 4 9 \%}$ \\
\hline
\end{tabular}

TABLE I: Labels matching results obtained by applying the NWA across the five labelers.

LHO). Overall, 413 labels were deleted from a total of 27683 manually labeled events (1.49\%). In no matching instance, there have been more than 5 iterations until convergence.

\section{E. Ground-Truth Limits of Agreement}

Provided the matched events among labelers, we compute the statistics of human labeling. The statistics are computed across labelers, i.e. given all matched labels for an event $\vec{l}_{i}$ by labeler $i$, we group all events into matrix form as

$$
L=\left(\vec{l}_{0}, \vec{l}_{1}, \ldots, \vec{l}_{N-1}\right) \in \mathbb{R}^{M \times N}
$$

where $N$ is the number of labelers, and then compute the ground-truth labels by averaging the rows of $L$. Furthermore, we compute the limits of agreement (LOA) as follows:

1) For each row $L_{i, \text { : }}$ of $L$, we compute the average value of the label $\mu_{i}=\mathrm{E}_{j}\left[L_{i, j}\right]$;

2) For each row $L_{i, \text { : }}$ of $L$, we compute $N$ error values as $e_{i, j}=\mu_{i}-L_{i, j}$, and collect these into an error vector $\vec{e}=\left(e_{i, j}\right) \forall(i, j) \in\{0, \ldots M-1\} \times\{0, \ldots, N-1\}$ with $M$ being the number of matched labels for the event of interest;

3) We compute the LOA as the 95-th percentile of $\vec{\epsilon}=$ $\left(\left|e_{k}\right|\right), k \in\{0,1, \ldots, M \cdot N-1\}$.

By applying this procedure, the LOA results illustrated Fig. 3 are obtained. The LOA in this work for HO, TO, HS, and FF are $72 \mathrm{~ms}, 16 \mathrm{~ms}, 24 \mathrm{~ms}$, and $80 \mathrm{~ms}$ respectively.

Summarizing, human labelers tend to agree well i.e., $\mathrm{LOA} \sim \mathcal{O}(20 \mathrm{~ms})$ for HS and TO, while lower accuracy and precision LOA $\sim \mathcal{O}(75 \mathrm{~ms})$ are achieved for $\mathrm{HO}$ and FF. This is due to the intrinsic difficulty that $\mathrm{HO}$ and FF come within their definitions and consequent difficulties for humans to decide whether an event of these types has occurred. As a consequence, the limits in terms of accuracy claims of automated systems are to be set around the identified LOA i.e., the LOA is the limit of accuracy for our system as it represents the intrinsic uncertainty in the ground-truth measurements. 

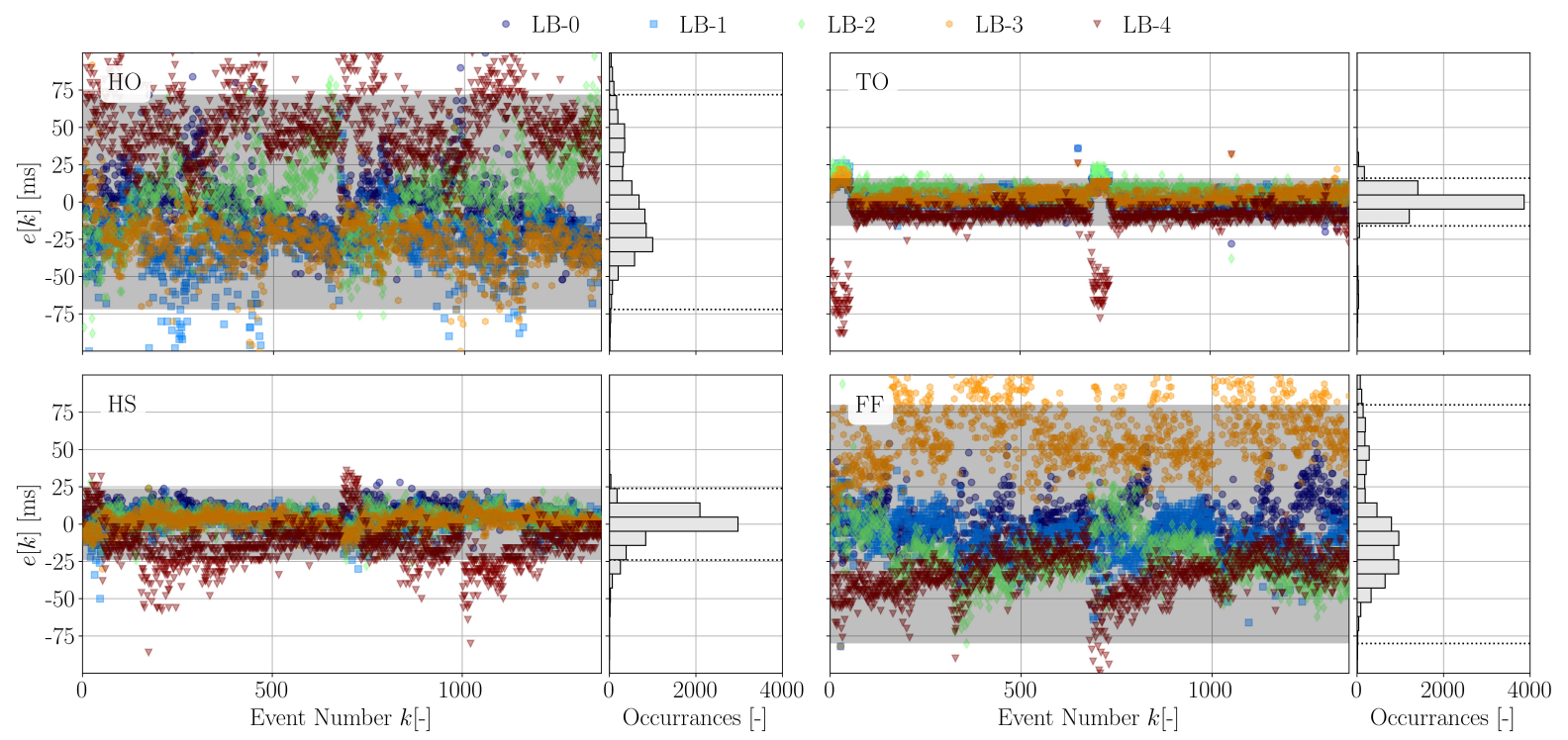

Fig. 3: LOA of labelers. The distributions for the four events HO, TO, HS, and FF as well as the LOA for the five labelers are depicted. The LOA in this work for HO, TO, HS, and FF are $72 \mathrm{~ms}, 16 \mathrm{~ms}, 24 \mathrm{~ms}$, and $80 \mathrm{~ms}$ respectively. In each subplot: the left plot shows all identified samples (coded by labeler LB) as deviation from the average label - the gray area is the LOA band; the right plot shows the distribution of all label errors - it can be understood as a projection of all samples of the left plot onto the $y$-axis. The dotted lines in the histogram mark the LOA band.

\section{Automated Gait Analysis Based on ML}

\section{A. Automatic Analysis Pipeline}

This section describes how our proposed gait analysis algorithm works. The first step is identifying potential strides within a WGAS recording by analyzing the recorded angular rate and acceleration raw data, and segmenting the data into regions of motion (ROM), which represent some continuous foot movement and potential strides. To classify whether a particular ROM constitutes a stride or not, its acceleration and angular rate are compared to those of a template stride, resulting in a number of similarity metrics. Together with the rescaled and resampled raw signals, these similarity metrics are fed to stepperNet, a convolutional neural network, deciding if the ROM represents a stride or not. Lastly, if a region has been identified as a stride, gait event detection algorithms are applied for intra-stride based detection of $\mathrm{HO}$, TO, HS, and FF. Any non-stride regions, on the other hand, are rejected for further analysis.

\section{B. Tools and Libraries}

The proposed gait stride detection and gait event detection algorithms are implemented in Python3. Further important tools include PyTorch, which was used as our machinelearning framework [55] and the dtw-python library as our dynamic time warping (DTW) library [56]. Lastly, our algorithms extensively use various toolboxes of the SciPy library [57].

\section{Data Segmentation}

Given a recording by the WGAS system, the raw data is first segmented into several ROM, the set of all ROM is denoted as $R_{M}=\left\{R_{M}^{i}: R_{M}^{i}\right.$ is ROM $\}$. Each ROM is defined by the start index $k_{0}^{i}$ and the end index $k_{f}^{i}$, in which the foot was non-stationary i.e., $R_{M}^{i}=\left(k_{0}^{i}, k_{f}^{i}\right)$. For the raw data segmentation, a set of four mean absolute signals, $a_{m}, \dot{a}_{m}, \omega_{m}, \dot{\omega}_{m}$ are constructed from $\vec{a}$ and $\vec{\omega}$. For a generic measurement taken along three axes $\vec{s}=\left(s_{x}, s_{y}, s_{z}\right)$, with sampling index $k$, its mean absolute computed by

$$
s_{m}[k]=\mathrm{E}_{l}\left[\left|s_{l}[k]\right|\right], l \in\{x, y, z\} .
$$

In a first step, the recording is segmented into general $R_{M}^{i}$ by scanning for coherent sequences that satisfy following conditions:

1) $f_{l p}(\dot{a}, 0.75 \mathrm{~Hz})>1 \mathrm{~m} / \mathrm{s}^{3}$;

2) $\Delta t^{i}=t\left[k_{f}^{i}\right]-t\left[k_{0}^{i}\right]>0.25 \mathrm{~s}$;

where $f_{l p}\left(x, f_{c}\right)$ denotes a low-pass filter with a cut-off frequency $f_{c}$ applied to $x$. These two conditions ensure that a resulting $R_{M}^{i}$ contains enough motion and the duration of the region is long enough to warrant further analysis. In the second segmentation step, each $R_{M}^{i}$ is split further by applying region-specific thresholds. Taking the example of a generic mean absolute signal from (2) on the interval of $R_{M}^{i}$, $s_{m}^{i}=\left\{s_{m}^{i}[k] \mid k \in\left[k_{0}^{i}, k_{f}^{i}\right]\right\}$ is given by

$$
T_{s_{m}}^{i}=b_{l}+\alpha\left(\mathrm{E}_{k^{\prime}}\left[s_{m}^{i}\left[k^{\prime}\right]\right]-b_{l}\right)
$$

where $k^{\prime}$ denotes the signal segment's running index, which will from here on denote a segment's running index. $\alpha$ is a tuning parameter and $b_{l}$ refers to the baseline of $s_{m}^{i}$. The 

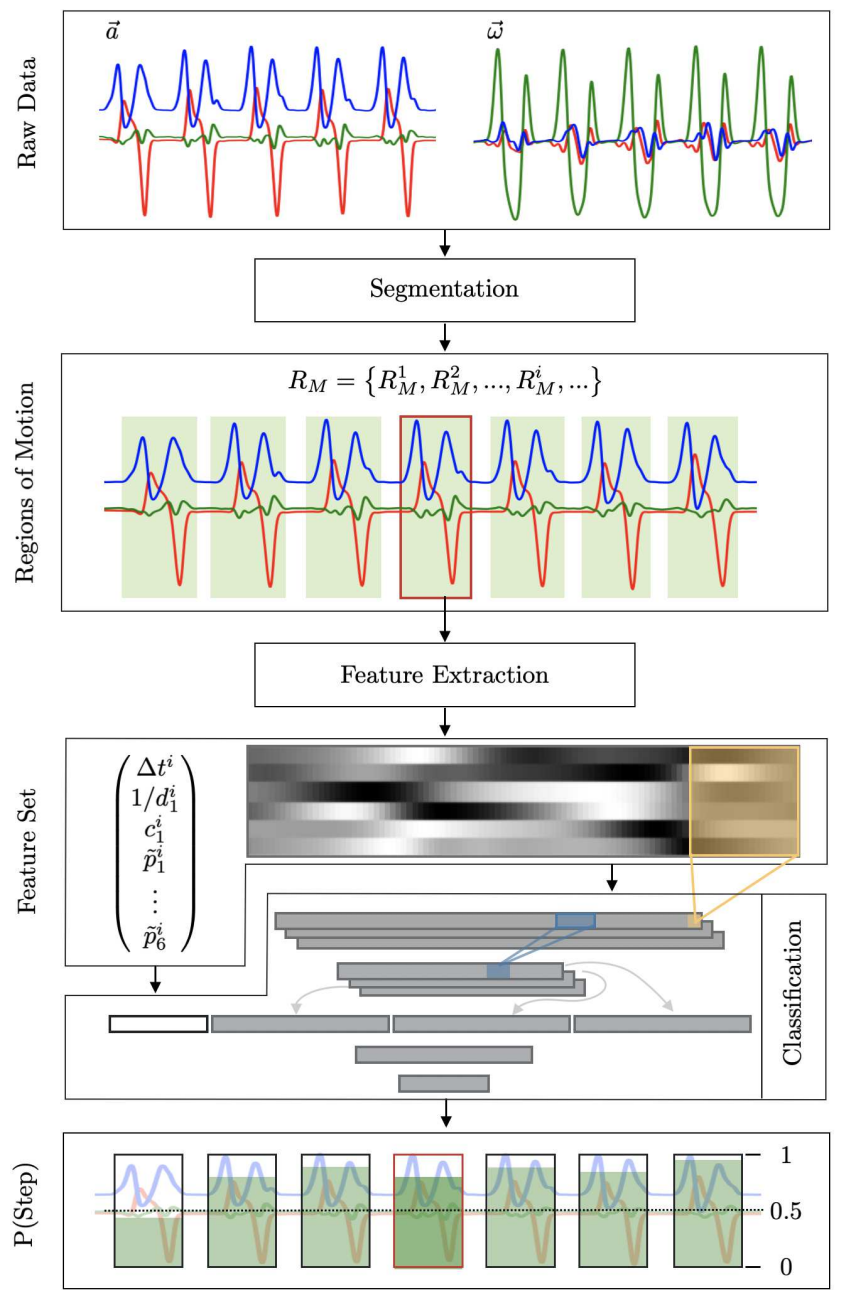

Fig. 4: Illustration of the WGAS's automated stride detection pipeline, which takes in raw WGAS data $(\vec{a}$ and $\vec{\omega})$, from which it detects gait strides. The figure outlines each performed operation and shows its respective result. First, the raw data is segmented into ROM, which constitutes candidate strides. Each ROM is further analyzed individually, by extracting features which are passed to stepperNet, whose architecture is detailed in Section IV-E Finally, stepperNet determines the probability of the passed features belonging to a stride i.e. $\mathrm{P}($ Stride $)=1$.

latter is estimated using iterative polynomial fitting [58]. By thresholding $a_{m}^{i}$ and $\omega_{m}^{i}$ against their respective thresholds, $T_{a_{m}}^{i}$ and $T_{\omega_{m}}^{i}$, they are binarized by

$$
\begin{aligned}
a_{b}^{i}\left[k^{\prime}\right] & =\left\{\begin{array}{l}
1, \text { if } a_{m}^{i}\left[k^{\prime}\right]>T_{a_{m}}^{i} \\
0, \text { else }
\end{array}\right. \\
\omega_{b}^{i}\left[k^{\prime}\right] & =\left\{\begin{array}{l}
1, \text { if } \omega_{m}^{i}\left[k^{\prime}\right]>T_{\omega_{m}}^{i} \\
0, \text { else }
\end{array}\right.
\end{aligned}
$$

Each $R_{M}^{i}$ detected in the initial segmentation is further divided into continuous regions which satisfy $f_{l p}\left(a_{b}^{i}+\omega_{b}^{i}, 4 \mathrm{~Hz}\right)>1.15$. Lastly, to ensure the segmentation procedure only yields relevant data segments, which represent candidate strides, any $R_{M}^{i}$ are discarded that do no meet $\mathrm{E}_{k^{\prime}}\left[\dot{a}_{m}^{i}\left[k^{\prime}\right]\right]>10 \mathrm{~m} / \mathrm{s}^{3}$ and $\mathrm{E}_{k^{\prime}}\left[\dot{\omega}_{m}^{i}\left[k^{\prime}\right]\right]>0.8 \mathrm{rad} / \mathrm{s}^{2}$.

\section{Feature Extraction}

After the data has been filtered to identify possible strides, each $R_{M}^{i}$ and the underlying IMU data, $\vec{a}^{i}$ and $\vec{\omega}^{i}$, is characterized by a set of features $F^{i}=\left\{\mathbf{M}^{i}, \mathbf{c}^{i}\right\}$, consisting of a signal mosaic and correlation scores, $\mathbf{M}^{i} \in \mathbb{R}^{100 \times 6}$ and $\mathbf{c}^{i} \in \mathbb{R}^{19}$, respectively. The former can be understood as a visual representation of the signals, while the latter is a measure of how similar the region's underlying signals are comparing to those of an average, healthy, template stride. To ease notation, the six IMU signals on $R_{M}^{i}$ are collectively referred to as $\zeta^{i}=\left(a_{x}^{i}, a_{y}^{i}, a_{z}^{i}, \omega_{x}^{i}, \omega_{y}^{i}, \omega_{z}^{i}\right) \in \mathbb{R}^{K^{i} \times 6}$ with $K^{i}$ being the number of time samples in $R_{M}^{i}$. We can then define the $j$-th column of the mosaic $\mathbf{M}_{:, j}^{i}$ to be

$$
\mathbf{M}_{:, j}^{i}=f_{\mathrm{RN}}\left(\zeta_{:, j}^{i}\right)
$$

where $f_{\mathrm{RN}}: \mathbb{R}^{K^{i} \times 6} \rightarrow[0,1]^{100 \times 6}$ is the resampling and normalizing function. Hence, each column vector of $\mathbf{M}^{i}$ each represents one channel of the IMU signals, which has been resampled to 100 data points in length and confined to $[0,1]$. The matrix can thus be understood as a grayscale image of the six IMU signals of $R_{M}^{i}$, as shown in Fig. 4.

The correlation scores, $\mathbf{c}^{i}$, are given by the comparison of $\zeta^{i}$ to the acceleration and angular rate of a template stride, $\hat{T}=\left(\hat{a}_{x}, \hat{a}_{y}, \hat{a}_{z}, \hat{\omega}_{x}, \hat{\omega}_{y}, \hat{\omega}_{z}\right)$, in terms of similarity. $\hat{T}$ is an average of 448 recorded strides taken by five healthy individuals and represents what a regular stride looks like in terms of acceleration and angular rate.

To account for temporal distortions, such as different walking speeds and/or slight time differences in their general gait pattern, DTW is applied to align each signal $\zeta_{j}^{i}$ onto its corresponding template $\hat{T}_{j}$. In short, DTW is a temporal transformation of $\zeta_{j}^{i}$, such that the cumulative distance of the resulting warped signal, $\tilde{\zeta}_{j}^{i}$ and $\hat{T}_{j}$ is minimized under some constraints. This can be understood as

$$
\tilde{\zeta}_{j}^{i}=\operatorname{DTW}\left(\zeta_{j}^{i}, \hat{T}_{j}\right)=\operatorname{argmin}_{\hat{\zeta}_{j}^{i} \in \mathcal{C}}\left\|\hat{\zeta}_{j}^{i}-\hat{T}_{j}\right\|
$$

where $\mathcal{C}$ denotes the set of all $\zeta_{j}^{i}$ which satisfy all constraints of the DTW problem. For a deeper understanding of DTW, we refer to [59], [60].

The similarity of $\tilde{\zeta}_{j}^{i}$ and $\hat{T}_{j}^{i}$ is characterized by three similarity metrics, namely the euclidean distance, Pearson's correlation coefficient, and the p-value of the correlation denoted by $d_{j}^{i}, c_{j}^{i}$ and $p_{j}^{i}$, respectively. As six signals are analyzed in this manner, a total of 18 metrics are computed, characterizing the ROM to the template stride. Due to the nature of bringing the signals into alignment using DTW, prior to comparing them, $p_{j}^{i}$ takes on very small values, thus in an effort to make them more easily interpretable, they are rescaled by $\bar{p}_{j}^{i}=0.1 \log p_{j}^{i}$. Finally, the vector $\mathbf{c}^{i}$ also holds the duration of $R_{M}^{i}, \Delta t^{i}=t\left[k_{f}^{i}\right]-t\left[k_{0}^{i}\right]$ and can be understood as

$$
\begin{aligned}
\mathbf{c}^{i} & =\left(\Delta t^{i}, c_{1}^{i}, d_{1}^{i}, p_{1}^{i}, \ldots, c_{6}^{i}, d_{6}^{i}, p_{6}^{i}\right) \\
& =\left(\Delta t^{i}, c_{a_{x}}^{i}, d_{a_{x}}^{i}, p_{a_{x}}^{i}, \ldots, c_{\omega_{z}}^{i}, d_{\omega_{z}}^{i}, p_{\omega_{z}}^{i}\right) .
\end{aligned}
$$




\section{E. Feature Classification}

Each feature set $F^{i}$ is then fed into a neural network, which is tasked with classifying if the corresponding $R_{M}^{i}$ represents a stride or not. The architecture of the proposed CNN, stepperNet is illustrated in Fig. 4, which accepts $F^{i}=$ $\left\{\mathbf{M}^{i}, \mathbf{c}^{i}\right\}$ as an input and outputs the probability of the feature set being a stride. It is composed of six total layers. The first is a convolutional layer, in which three kernels (with dimensions of $10 \times 6$ ), a step size of 1 , and zero padding are applied to $\mathbf{M}^{i}$. The resulting layer $(91 \times 1 \times 3)$, applies a max pooling kernel $(3 \times 1)$, to compress the dimensionality. The three reduced feature maps are then concatenated into a single vector $(90 \times 1)$, to which $\mathbf{c}^{i}$ is appended. The joined vector is then passed through two fully connected layers with 30 and 15 neurons each. Finally, the step probability is given by a single neuron activated by the soft-max function. Apart from the latter, each neuron employs the rectified linear activation function. Thus, any feature set with a stepperNet output greater than 0.5, is considered to be a stride, whereas if the stride probability lower than 0.5 is assumed to be some other foot motion.

Our dataset consisted of 4014 labeled feature sets, of which 1888 represented strides, while the rest characterized some other form of foot movement. Note, that the dataset used to train and test stepperNet is completely separate from the data collected for the validation of the gait-events detection algorithm. All data were collected in-house by five healthy subjects who were tasked with imitating various gait patterns and performing a variety of non-stride foot motions. For training stepperNet, the collected data were randomly split into a training dataset (3493 features), with which the network's parameters were optimized and a test set (521) which was used to monitor stepperNet's performance in training.

stepperNet was trained by stochastic gradient descent [61] over 7 epochs, with the learning rate and momentum set to 0.015 and 0.75 respectively. Due to the small network size and the limited number of training examples, the performance of the trained network is highly dependent on the initially chosen weights and biases. To maximize the stride classification capabilities of stepperNet, the training procedure was repeated multiple times, each time with different random initial internal parameters, and the highest scoring network was saved and deployed. stepperNet's performance at the conclusion of the training procedure on the training and validation data are reported in Fig. 5

\section{F. Gait Event Detection}

For an $R_{M}^{i}$, which has been determined to constitute a stride, the four gait events are identified by event-specific features in the recorded acceleration and angular rate on the interval specified in $R_{M}^{i}$. The correspondence of these features to the occurrence of the event was established experimentally by comparing WGAS recordings to the manual identified events of an accompanying motion capture system measurement. For reference, Fig. 6 depicts the raw acceleration and angular rate over a stride recorded by WGAS's IMU, together with the location of the gait events.

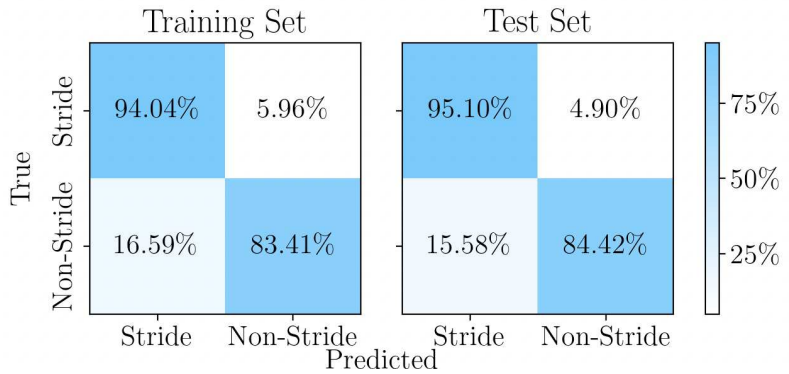

Fig. 5: The performance of stepperNet at the conclusion of its training on the training dataset and the separate test dataset. The residual loss on the two datasets is $4.26 \mathrm{e}-3$ and $4.02 \mathrm{e}-3$ respectively.

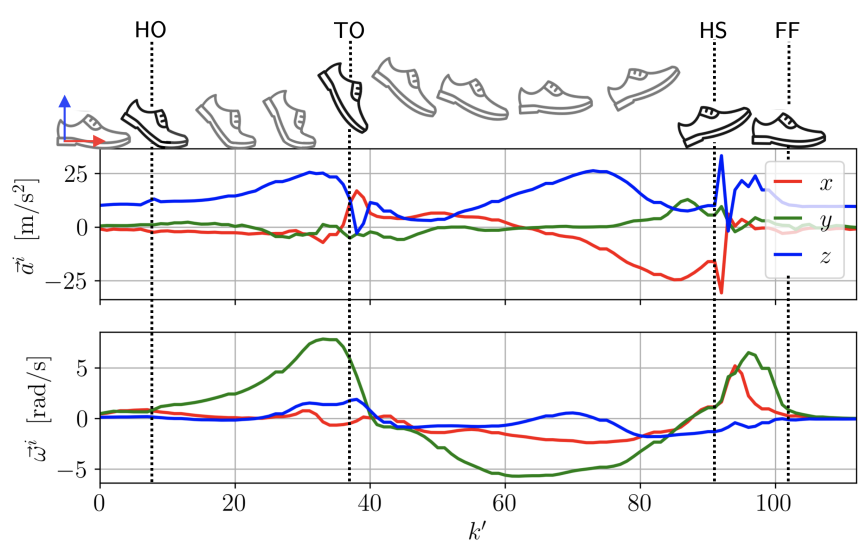

Fig. 6: Plot of the measured acceleration and angular rate over the course of a stride. Depicted are the four gait events, HO, TO, HS, and FF which were identified by manual analysis of the motion capture system data.

The start and end of a stride are defined by the $\mathrm{HO}$ and $\mathrm{FF}$, respectively and the foot can be assumed to be moving there between. As both events are the result of changes in the foot's orientation, $\mathrm{HO}$ and $\mathrm{FF}$ are detected by taking the first and last instance where $\left|\hat{\omega}^{i}\right| / \sigma_{\left|\hat{\omega}^{i}\right|}>0.5$, where $\sigma_{\left|\hat{\omega}^{i}\right|}$ is the standard deviation of $\left|\hat{\omega}^{i}\right|$.

The HS event is most easily identifiable in the accelerometer data, as the event resembles an impulse that manifests itself in an abrupt change in the measured acceleration. This impact is markedly experienced in the $z$-direction, which is the reason that the HS event is determined by the location of the most prominent peak in $\dot{a}_{z}^{i}$.

In comparable gait systems, the TO event is identified by the first peak in $\omega_{y}$ [43], [44]. Although a good initial guess, by comparing WGAS's measurements to the recordings of the motion capture system we find that the first instance where $a_{x}^{i}=a_{z}^{i}$ to be a better indication for the occurrence of the event.

\section{VALIDATION RESULTS}

\section{A. Data Synchronization}

1) Vibration Sync: For our work, a validation dataset consists of two WGAS measurements (left and right) with a corresponding motion capture system recording, each of which 
is logged with its own internal clocks. In order to be able to compare the events labeled manually to the events detected by the gait event detection algorithm, we first ensure that they are temporally synced to one another. To this end, each WGAS is set to initiate a vibration sequence once they are turned on, vibrating for $1 \mathrm{~s}$, a $1 \mathrm{~s}$ pause, and a second vibration of $1 \mathrm{~s}$.

As the experiment starts with the user standing on the treadmill of the motion capture system the vibration sequence is also picked up by the treadmill's integrated force plate. This allows for the temporal syncing between the data collected by the two systems.

2) Hard Cropping: A couple of seconds after the vibration sequence, the treadmill was turned on and the subject started to walk. In order for the treadmill to reach the preset speed and to allow subjects to settle into their preferred gait at the imposed speed, only motion capture system data that was collected after the individual had signaled the start of the experiment by hand, was manually labeled for gait events. This signal was clearly observable in the motion capture system recording, allowing the exact establishment of the experiment's start time $t_{0}$.

\section{B. Events Matching}

As for our analysis on the LOA of human labelers, the comparison of WGAS events and manually-labeled events cannot occur immediately. Again, there is no a priori guarantee that there is a one-to-one correspondence between the events detected by the two approaches. Therefore, we use the NWA to match WGAS events to manually-labeled events. The discarded events are considered to be outliers.

\section{Results}

To compute the performance of our gait event detection algorithms, the timestamp of the $i$-th gait event, $G$, detected by our algorithm, $t_{N}[G]_{i}$, is compared to the timestamp of the event identified manually in the motion capture system data, $t_{V}[G]_{i}$. Note that only events that have been matched using the NWA are analyzed and that for our comparison, $t_{V}[G]_{i}$ is taken from the average timestamp that all labelers assigned the event.

To quantify the accuracy of our gait event detection algorithms, the error, $e[G]_{i}$, for a given event pair, $t_{V}[G]_{i}$ and $t_{N}[G]_{i}$, is defined as

$$
e[G]_{i}=t_{V}[G]_{i}-t_{N}[G]_{i} .
$$

For our overall errors, we define the average error as $\mathrm{E}_{i}[e[G]]$, the mean absolute error as $\mathrm{MAE}=\mathrm{E}_{i}[|e[G]|]$ and the root mean squared error as RMSE $=\sqrt{\mathrm{E}_{i}\left[e[G]^{2}\right]}$, for the event type $G$. Further we also compute the 95 -th percentile of $e[G]_{i}$, which is referred to as the limit of agreement and is denoted by LOA.

In total, the detected gait events for roughly 1350 strides are compared to the labeled ground truth. This number differs from event to event, as any manually labeled event not identified by all labelers is omitted. The overall error metrics for our detected $\mathrm{HO}, \mathrm{TO}, \mathrm{HS}$ and $\mathrm{FF}$, averaged across all subjects and treadmill speeds are presented in Table II The average error for every detected event is smaller than WGAS's

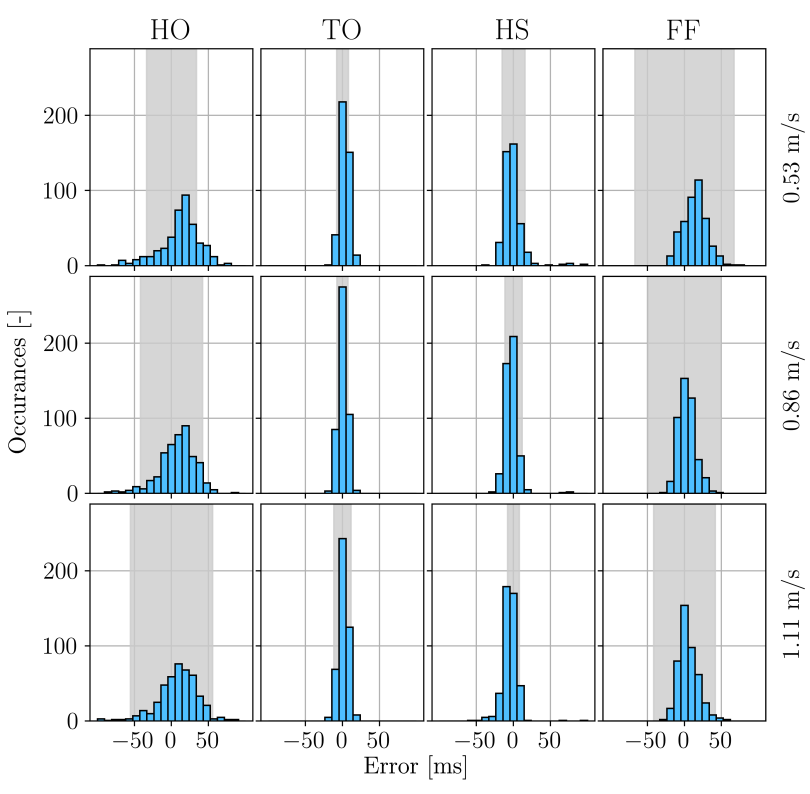

Fig. 7: Error histograms for the event detection errors grouped by the set treadmill speed and event type. The histograms are plotted with a bin size of $10 \mathrm{~ms}$, and for comparison, the uncertainty bounds of the manual labeled events are shown in gray.

sampling time, indicating our syncing method to be sufficient as only a very little bias remains.

The error metrics are also presented, for each event averaged across all subjects but grouped by treadmill speed, which is shown in Fig. 7. The algorithm's event detection error can be seen to slightly decrease for increasing speeds, which can be understood by the higher acceleration and angular rates, leading to more pronounced, unambiguous features within the data. As this effect is minor, the event detection algorithm can be regarded to be invariant and robust towards differences in gait speed.

Generally, it should be stated, that it is difficult to discern if an error is the result of faulty event detection of the algorithm or due to the subjective nature of manual labeling. For the $\mathrm{HO}$ and FF, this is especially true, as the events are not definitively identifiable in the motion capture system data by eye introducing an error in the ground truth itself. An infinite number of labelers may average out every individual labeling error, but due to time and cost constraints, the limited number of labelers in this study, the ground truth may still be imperfect.

Comparing the distribution, in terms of LOA, of the error of our detected events, to those of ground truth, found in Table ?? and Table [I] respectively, suggests that the algorithms are generally more consistent in their predictions. An exception is the HS event, for which the error of the detected LOA is greater compared to that of the manual labels. 


\begin{tabular}{|c|c|c|c|c|c|c|}
\hline Event & $\begin{array}{c}\text { Mean } \\
{[\mathrm{ms}]}\end{array}$ & $\begin{array}{c}\text { STD } \\
{[\mathrm{ms}]}\end{array}$ & $\begin{array}{c}\text { MAE } \\
{[\mathrm{ms}]}\end{array}$ & $\begin{array}{c}\text { RMSE } \\
{[\mathrm{ms}]}\end{array}$ & $\begin{array}{c}\text { P }_{95} \\
{[\mathrm{~ms}]}\end{array}$ & N [-] \\
\hline HO & 9 & 29 & 24 & 31 & 60 & 1338 \\
\hline TO & 2 & 7 & 5 & 7 & 10 & 1347 \\
\hline HS & -2 & 16 & 9 & 16 & 20 & 1355 \\
\hline FF & 9 & 34 & 13 & 36 & 30 & 1349 \\
\hline
\end{tabular}

TABLE II: Error metrics for gait event detection

\section{DISCUSSION}

Manual labeling of four gait events i.e., HO, TO, HS, and $\mathrm{FF}$, are evaluated in this paper, as they are essential for analyzing temporal gait parameters, such as stride time, swing time, stance time, single and double support time. The labels of the main events for gait segmentation, HS and TO, exhibit a remarkable consistency among different labelers with the uncertainty within two samples. However, the quality of $\mathrm{HO}$ and FF labels is much lower. This is due to the inherent challenges in observing $\mathrm{HO}$ and $\mathrm{FF}$ in optical data from motion capture systems, and different perspectives among different labelers. Gait is described as a repetitive, cyclic movement. The gait cycles are in most cases delimited by the HS event, which is considered of paramount functional importance marking the shift from flexors to anti-gravity muscles and is also reliably detectable [62]. To calculate gait parameters for each gait cycle, the movement data are interpolated from HS to the next ipsilateral HS. Errors in HS detection lead to normalization artefacts that can affect the whole gait cycle and hence all calculated parameters. TO delineates the two main phases of gait: stance and swing. Kinematic parameters are frequently reported for these phases separately, hence errors in TO detection affect not only values at TO but also parameters calculated for the phases, e.g. "knee range of motion during swing". At both time points, parameters are frequently reported that are in phases of rapid change, such as sagittal ankle and knee angle at TO (both in the range of $100-300 \%$ in dependence on walking speed), or foot elevation angle at HS [63]. Concerning the gait events $\mathrm{HO}$ and FF, there is a growing interest in the importance of these phases that depict a first reaction to a state change from swing-to-stance [64] and the preparatory phase from stanceto-swing [65]-[67]. Detecting these additional gait events also allows the calculation of parameters related to push off and initial contact. These events do not correspond with traditional clinical phases of gait, however present a technical mean to acquire information about these critical states. Overall, more detailed segmentation of the gait cycle and calculation of movement parameters that provide a relationship to typical recovery patterns in clinical populations allow clinicians to make more objective and detailed assessments of the deviation in pathological gait and the improvements in rehabilitation progress. Furthermore, gait event detection is also critical for applications in which gait events are served to trigger assistive devices, as well as when considering the use of orthotic or therapeutic interventions, particularly in functional electrical stimulation [68].

The presented results on the limits of detection for key gait events also indicate that the quality of manual labels for gait events generated from optical motion capture systems is significantly dependent on the labeler's personal perspective and experience. Low consistency between labelers can have a direct impact on gait event detection reliability. This affects the estimation accuracy of spatio-temporal gait parameters, as well as the medical decision-making and therapy by clinicians. On the other hand, the results suggest that this uncertainty represents the accuracy limit of human gait labeling based on motion capture data, and it determines the upper limit for any validation study which considers the motion capture system as the gold standard.

A template-based DTW and CNN architectures were employed to identify strides and reject non-strides from walking signals automatically. A rule-based gait event detecting algorithm was adopted to segment the identified strides into subphases for spatial and temporal gait parameters estimation. Note that the CNN is only used for identifying a valid stride and eliminating non-stride data from the dataset that was acquired over a long walking period. Gait events are afterwards detected within the region of this identified stride by searching for specific signal characteristics. Opposed to the commonly used approach in the literature, which straightly scans the entire walking recording to identify the gait events and then employs the gait event to define a stride, CNN eliminates all non-stride data and only keeps stride data for further gait event detection. This approach not only increases the computational efficiency and saves computation time, but also shows potential for adapting this approach to pathological gait where some gait events may be missing. It may become a useful tool for achieving personalized gait analysis.

This work is based on a limited number and type of validation subjects, but it still provides important insight on analyzing pathological gait in a robust and efficient way. Furthermore, the selection of manual labeling of the motion capture data as the ground truth induces caveats. Vertical ground reaction force, specifically, was not used in this study due to a large amount of foot strikes that loaded both force plates. These events would have needed to be excluded which would have introduced a bias into the data set. Therefore group-based manual labeling was performed, which is also representative of how pathological gait is frequently segmented. Pathological gait has to be addressed in the future for assessing the validity of the proposed method for pathological subjects. In this study, the stride template was generated by averaging all strides. While for pathological gait, the strides will not be as homogeneous, new methodologies have to be developed for optimizing the stride template, such as generating template from kernel density estimation or Gaussian mixture model, constructing a library of stride templates from different pathologies or experimental conditions. In this work, stepperNet is only validated on treadmill walking data, one subject's stride is likely to be identical during the same speed session. As the stepperNet is primarily designed for identifying walking strides and eliminating the non-walking strides data from long-term free walking data to reduce the 
computational cost, the performance of stepperNet on longterm free walking in the real-world must be validated. Future work will focus on investigating the detection accuracy of stepperNet in various conditions, including variable speed walking, free-range walking, and various clinical populations.

\section{CONCLUSION}

We described a method to estimate and analyze the uncertainty in manual labeling of human walking data from motion capture systems, which are commonly used as a benchmark. Human manual labeling uncertainty was assessed by measuring inter-labeler inconsistencies. The limits of detection for key gait events, $\mathrm{HO}, \mathrm{TO}, \mathrm{HS}$, and FF have been estimated as $72 \mathrm{~ms}, 16 \mathrm{~ms}, 22 \mathrm{~ms}$, and $80 \mathrm{~ms}$. Those inherent label uncertainty of human gait labeling based on motion capture data is the accuracy limit for any validation research using this technology.

We also present a novel CNN-based algorithm that is able to detect key gait events with sub-10ms precision and auxiliary gait events with sub-25ms precision in healthy, fixed speed treadmill walking. The algorithm provides an improvement on current solutions in that it allows the accurate processing of long continuous walking data streams. Clinically, the accurate detection of these events reduce distortion effects when calculating gait parameters, providing a gateway to reliable parameter calculation that are lower than the minimal clinically important difference. Achieving this accuracy in gait parameters is paramount for empowering clinical decision making based on mobile gait analysis.

\section{ACKNOWLEDGMENT}

This project has received funding from the European Union's Horizon 2020 Research and Innovation program under the Marie Sklodowska-Curie grant agreement No. 764977.

\section{CONFLICT OF INTEREST}

B.J.N. and S.P. are shareholders in Magnes AG.

\section{REFERENCES}

[1] S. Del Din, A. Godfrey, B. Galna, S. Lord, and L. Rochester, "Freeliving gait characteristics in ageing and parkinson's disease: impact of environment and ambulatory bout length," Journal of neuroengineering and rehabilitation, vol. 13, no. 1, pp. 1-12, 2016.

[2] C. L. Richards, F. Malouin, and C. Dean, "Gait in stroke: assessment and rehabilitation," Clinics in geriatric medicine, vol. 15, no. 4, pp. 833-856, 1999.

[3] C. Selves, G. Stoquart, and T. Lejeune, "Gait rehabilitation after stroke: review of the evidence of predictors, clinical outcomes and timing for interventions," Acta Neurologica Belgica, vol. 120, no. 4, pp. 783-790, 2020.

[4] J. J. Eng and P.-F. Tang, "Gait training strategies to optimize walking ability in people with stroke: a synthesis of the evidence," Expert review of neurotherapeutics, vol. 7, no. 10, pp. 1417-1436, 2007.

[5] A. Lees, "When did ray kennedy's parkinson's disease begin?" Movement disorders: official journal of the Movement Disorder Society, vol. 7, no. 2, pp. 110-116, 1992.

[6] L. Brognara, P. Palumbo, B. Grimm, and L. Palmerini, "Assessing gait in parkinson's disease using wearable motion sensors: a systematic review," Diseases, vol. 7, no. 1, p. 18, 2019.

[7] R. Mc Ardle, B. Galna, P. Donaghy, A. Thomas, and L. Rochester, "Do alzheimer's and lewy body disease have discrete pathological signatures of gait?" Alzheimer's \& Dementia, vol. 15, no. 10, pp. 1367-1377, 2019.
[8] L. Z. Gras, S. F. Kanaan, J. M. McDowd, Y. M. Colgrove, J. Burns, and P. S. Pohl, "Balance and gait of adults with very mild alzheimer's disease," Journal of geriatric physical therapy (2001), vol. 38, no. 1, p. 1, 2015.

[9] M. Hoskovcová, O. Ulmanová, O. Šprdlík, T. Sieger, J. Nováková, R. Jech, and E. Růžička, "Disorders of balance and gait in essential tremor are associated with midline tremor and age," The Cerebellum, vol. 12 , no. 1 , pp. 27-34, 2013.

[10] H. Stolze, G. Petersen, J. Raethjen, R. Wenzelburger, and G. Deuschl, "The gait disorder of advanced essential tremor," Brain, vol. 124, no. 11, pp. 2278-2286, 2001.

[11] B. Balaban and F. Tok, "Gait disturbances in patients with stroke," $P M \& R$, vol. 6, no. 7, pp. 635-642, 2014.

[12] H. Jacobi, T.-K. Hauser, P. Giunti, C. Globas, P. Bauer, T. SchmitzHübsch, L. Baliko, A. Filla, C. Mariotti, M. Rakowicz et al., "Spinocerebellar ataxia types 1, 2, 3 and 6: the clinical spectrum of ataxia and morphometric brainstem and cerebellar findings," The Cerebellum, vol. 11, no. 1, pp. 155-166, 2012.

[13] L. Nürnberger, C. Klein, S. Baudrexel, J. Roggendorf, M. Hildner, S. Chen, J.-S. Kang, R. Hilker, and J. Hagenah, "Ultrasound-based motion analysis demonstrates bilateral arm hypokinesia during gait in heterozygous pink1 mutation carriers," Movement Disorders, vol. 30, no. 3, pp. 386-392, 2015.

[14] M. Pistacchi, M. Gioulis, F. Sanson, E. De Giovannini, G. Filippi, F. Rossetto, and S. Z. Marsala, "Gait analysis and clinical correlations in early parkinson's disease," Functional neurology, vol. 32, no. 1, p. 28, 2017.

[15] J. A. DeLisa, Gait analysis in the science of rehabilitation. Diane Publishing, 1998, vol. 2.

[16] K. Kong and M. Tomizuka, "A gait monitoring system based on air pressure sensors embedded in a shoe," IEEE/ASME Transactions on mechatronics, vol. 14, no. 3, pp. 358-370, 2009.

[17] X. Meng, H. Yu, and M. P. Tham, "Gait phase detection in able-bodied subjects and dementia patients," in 2013 35th Annual International Conference of the IEEE Engineering in Medicine and Biology Society (EMBC). IEEE, 2013, pp. 4907-4910.

[18] C. M. Senanayake and S. A. Senanayake, "Computational intelligent gait-phase detection system to identify pathological gait," IEEE Transactions on Information Technology in Biomedicine, vol. 14, no. 5, pp. 1173-1179, 2010.

[19] A. Atrsaei, M. F. Corrà, F. Dadashi, N. Vila-Chã, L. Maia, B. Mariani, W. Maetzler, and K. Aminian, "Gait speed in clinical and daily living assessments in parkinson's disease patients: performance versus capacity," npj Parkinson's Disease, vol. 7, no. 1, pp. 1-11, 2021.

[20] R. Z. U. Rehman, S. Del Din, Y. Guan, A. J. Yarnall, J. Q. Shi, and L. Rochester, "Selecting clinically relevant gait characteristics for classification of early parkinson's disease: A comprehensive machine learning approach," Scientific reports, vol. 9, no. 1, pp. 1-12, 2019.

[21] L. Palmerini, L. Rocchi, S. Mazilu, E. Gazit, J. M. Hausdorff, and L. Chiari, "Identification of characteristic motor patterns preceding freezing of gait in parkinson's disease using wearable sensors," Frontiers in neurology, vol. 8, p. 394, 2017.

[22] M. Mancini, B. R. Bloem, F. B. Horak, S. J. Lewis, A. Nieuwboer, and J. Nonnekes, "Clinical and methodological challenges for assessing freezing of gait: Future perspectives," Movement Disorders, vol. 34, no. 6, pp. 783-790, 2019.

[23] M. Heijmans, J. G. Habets, C. Herff, J. Aarts, A. Stevens, M. L. Kuijf, and P. L. Kubben, "Monitoring parkinson's disease symptoms during daily life: a feasibility study," NPJ Parkinson's disease, vol. 5, no. 1 , pp. 1-6, 2019.

[24] C. L. Pulliam, D. A. Heldman, E. B. Brokaw, T. O. Mera, Z. K. Mari, and M. A. Burack, "Continuous assessment of levodopa response in parkinson's disease using wearable motion sensors," IEEE Transactions on Biomedical Engineering, vol. 65, no. 1, pp. 159-164, 2017.

[25] E. Warmerdam, J. M. Hausdorff, A. Atrsaei, Y. Zhou, A. Mirelman, K. Aminian, A. J. Espay, C. Hansen, L. J. Evers, A. Keller et al., "Long-term unsupervised mobility assessment in movement disorders," The Lancet Neurology, vol. 19, no. 5, pp. 462-470, 2020.

[26] V. V. Shah, J. McNames, M. Mancini, P. Carlson-Kuhta, R. I. Spain, J. G. Nutt, M. El-Gohary, C. Curtze, and F. B. Horak, "Laboratory versus daily life gait characteristics in patients with multiple sclerosis, parkinson's disease, and matched controls," Journal of neuroengineering and rehabilitation, vol. 17, no. 1, pp. 1-12, 2020.

[27] S. Mihradi, T. Dirgantara, A. I. Mahyuddin et al., "Development of an optical motion-capture system for 3d gait analysis," in 2011 2nd International Conference on Instrumentation, Communications, Information Technology, and Biomedical Engineering. IEEE, 2011, pp. 391-394. 
[28] B. Carse, B. Meadows, R. Bowers, and P. Rowe, "Affordable clinical gait analysis: An assessment of the marker tracking accuracy of a new low-cost optical 3d motion analysis system," Physiotherapy, vol. 99, no. 4, pp. 347-351, 2013.

[29] H. Yu, D.-h. Wang, C.-J. Yang, and K.-M. Lee, “A walking monitoring shoe system for simultaneous plantar-force measurement and gait-phase detection," in 2010 IEEE/ASME International Conference on Advanced Intelligent Mechatronics. IEEE, 2010, pp. 207-212.

[30] I. Papavasileiou, W. Zhang, and S. Han, "Real-time data-driven gait phase detection using ground contact force measurements: Algorithms, platform design and performance," Smart Health, vol. 1, pp. 34-49, 2017.

[31] J. Wu, K. Kuruvithadam, A. Schaer, R. Stoneham, G. Chatzipirpiridis, C. A. Easthope, G. Barry, J. Martin, S. Pané, B. J. Nelson et al., "An intelligent in-shoe system for gait monitoring and analysis with optimized sampling and real-time visualization capabilities," Sensors, vol. 21 , no. 8 , p. 2869, 2021.

[32] H. Zhang, Y. Guo, and D. Zanotto, "Accurate ambulatory gait analysis in walking and running using machine learning models," IEEE Transactions on Neural Systems and Rehabilitation Engineering, vol. 28, no. 1, pp. 191-202, 2019.

[33] C. Tunca, N. Pehlivan, N. Ak, B. Arnrich, G. Salur, and C. Ersoy, "Inertial sensor-based robust gait analysis in non-hospital settings for neurological disorders," Sensors, vol. 17, no. 4, p. 825, 2017.

[34] R. K. Laughman, L. J. Askew, R. R. Bleimeyer, and E. Y. Chao, "Objective clinical evaluation of function: Gait analysis," Physical therapy, vol. 64, no. 12, pp. 1839-1845, 1984.

[35] V. Robles-García, Y. Corral-Bergantiños, N. Espinosa, M. A. Jácome, C. García-Sancho, J. Cudeiro, and P. Arias, "Spatiotemporal gait patterns during overt and covert evaluation in patients with parkinson's disease and healthy subjects: is there a hawthorne effect?" Journal of applied biomechanics, vol. 31, no. 3, pp. 189-194, 2015.

[36] J. Kaye, N. Mattek, H. Dodge, T. Buracchio, D. Austin, S. Hagler, M. Pavel, and T. Hayes, "One walk a year to 1000 within a year: continuous in-home unobtrusive gait assessment of older adults," Gait \& posture, vol. 35, no. 2, pp. 197-202, 2012.

[37] R. Caldas, M. Mundt, W. Potthast, F. B. de Lima Neto, and B. Markert, "A systematic review of gait analysis methods based on inertial sensors and adaptive algorithms," Gait \& posture, vol. 57, pp. 204-210, 2017.

[38] D. Kobsar, J. M. Charlton, C. T. Tse, J.-F. Esculier, A. Graffos, N. M Krowchuk, D. Thatcher, and M. A. Hunt, "Validity and reliability of wearable inertial sensors in healthy adult walking: a systematic review and meta-analysis," Journal of neuroengineering and rehabilitation, vol. 17 , pp. 1-21, 2020 .

[39] M. Lempereur, F. Rousseau, O. Rémy-Néris, C. Pons, L. Houx, G. Quellec, and S. Brochard, "A new deep learning-based method for the detection of gait events in children with gait disorders: Proof-of-concept and concurrent validity," Journal of biomechanics, vol. 98, p. 109490, 2020.

[40] Ł. Kidziński, S. Delp, and M. Schwartz, "Automatic real-time gait event detection in children using deep neural networks," PloS one, vol. 14, no. 1, p. e0211466, 2019.

[41] W.-C. Hsu, T. Sugiarto, Y.-J. Lin, F.-C. Yang, Z.-Y. Lin, C.-T. Sun, C.-L. Hsu, and K.-N. Chou, "Multiple-wearable-sensor-based gait classification and analysis in patients with neurological disorders," Sensors, vol. 18 , no. 10 , p. $3397,2018$.

[42] V. Agostini, G. Balestra, and M. Knaflitz, "Segmentation and classification of gait cycles," IEEE Transactions on Neural Systems and Rehabilitation Engineering, vol. 22, no. 5, pp. 946-952, 2013.

[43] H. Zhao, Z. Wang, S. Qiu, Y. Shen, and J. Wang, "Imu-based gait analysis for rehabilitation assessment of patients with gait disorders," in 2017 4th International Conference on Systems and Informatics (ICSAI). IEEE, 2017, pp. 622-626.

[44] S. Zhu, H. Anderson, and Y. Wang, "A real-time on-chip algorithm for imu-based gait measurement," in Pacific-Rim Conference on Multimedia. Springer, 2012, pp. 93-104

[45] L. Liu, H. Wang, H. Li, J. Liu, S. Qiu, H. Zhao, and X. Guo, "Ambulatory human gait phase detection using wearable inertial sensors and hidden markov model," Sensors, vol. 21, no. 4, p. 1347, 2021.

[46] T. Zhen, L. Yan, and P. Yuan, "Walking gait phase detection based on acceleration signals using lstm-dnn algorithm," Algorithms, vol. 12 no. 12 , p. 253, 2019.

[47] M. Ullrich, A. Küderle, J. Hannink, S. Del Din, H. Gaßner, F. Marxreiter, J. Klucken, B. M. Eskofier, and F. Kluge, "Detection of gait from continuous inertial sensor data using harmonic frequencies," IEEE Journal of Biomedical and Health Informatics, vol. 24, no. 7, pp. 1869 1878,2020
[48] A. Hickey, S. Del Din, L. Rochester, and A. Godfrey, "Detecting freeliving steps and walking bouts: validating an algorithm for macro gait analysis," Physiological measurement, vol. 38, no. 1, p. N1, 2016.

[49] L. Oudre, R. Barrois-Müller, T. Moreau, C. Truong, A. Vienne-Jumeau, D. Ricard, N. Vayatis, and P.-P. Vidal, "Template-based step detection with inertial measurement units," Sensors, vol. 18, no. 11, p. 4033, 2018.

[50] J. Barth, C. Oberndorfer, C. Pasluosta, S. Schülein, H. Gassner, S. Reinfelder, P. Kugler, D. Schuldhaus, J. Winkler, J. Klucken et al., "Stride segmentation during free walk movements using multi-dimensional subsequence dynamic time warping on inertial sensor data," Sensors, vol. 15, no. 3, pp. 6419-6440, 2015.

[51] C. F. Martindale, V. Christlein, P. Klumpp, and B. M. Eskofier, "Wearables-based multi-task gait and activity segmentation using recurrent neural networks," Neurocomputing, vol. 432, pp. 250-261, 2021.

[52] A. Barre and S. Armand, "Biomechanical toolkit: Open-source framework to visualize and process biomechanical data," Computer methods and programs in biomedicine, vol. 114, no. 1, pp. 80-87, 2014.

[53] H. T. T. Vu, D. Dong, H.-L. Cao, T. Verstraten, D. Lefeber, B. Vanderborght, and J. Geeroms, "A review of gait phase detection algorithms for lower limb prostheses," Sensors, vol. 20, no. 14, p. 3972, 2020.

[54] S. B. Needleman and C. D. Wunsch, "A general method applicable to the search for similarities in the amino acid sequence of two proteins," Journal of Molecular Biology, vol. 48, pp. 443-453, 1970.

[55] A. Paszke, S. Gross, F. Massa, A. Lerer, J. Bradbury, G. Chanan, T. Killeen, Z. Lin, N. Gimelshein, L. Antiga et al., "Pytorch: An imperative style, high-performance deep learning library," arXiv preprint arXiv:1912.01703, 2019.

[56] T. Giorgino et al., "Computing and visualizing dynamic time warping alignments in r: the dtw package," Journal of statistical Software, vol. 31 , no. 7 , pp. 1-24, 2009

[57] P. Virtanen, R. Gommers, T. E. Oliphant, M. Haberland, T. Reddy, D. Cournapeau, E. Burovski, P. Peterson, W. Weckesser, J. Bright et al., "Scipy 1.0: fundamental algorithms for scientific computing in python," Nature methods, vol. 17, no. 3, pp. 261-272, 2020.

[58] F. Gan, G. Ruan, and J. Mo, "Baseline correction by improved iterative polynomial fitting with automatic threshold," Chemometrics and Intelligent Laboratory Systems, vol. 82, no. 1-2, pp. 59-65, 2006.

[59] D. J. Berndt and J. Clifford, "Using dynamic time warping to find patterns in time series." in KDD workshop, vol. 10, no. 16. Seattle, WA, USA:, 1994, pp. 359-370.

[60] M. Müller, "Dynamic time warping," Information retrieval for music and motion, pp. 69-84, 2007.

[61] L. Bottou, "Large-scale machine learning with stochastic gradient descent," in Proceedings of COMPSTAT'2010. Springer, 2010, pp. 177186.

[62] M. Burnfield, "Gait analysis: normal and pathological function," Journal of Sports Science and Medicine, vol. 9, no. 2, p. 353, 2010.

[63] B. F. Mentiplay, M. Banky, R. A. Clark, M. B. Kahn, and G. Williams, "Lower limb angular velocity during walking at various speeds," Gait \& posture, vol. 65, pp. 190-196, 2018.

[64] K. J. Nolan and M. Yarossi, "Preservation of the first rocker is related to increases in gait speed in individuals with hemiplegia and afo," Clinical Biomechanics, vol. 26, no. 6, pp. 655-660, 2011.

[65] S. A. Roelker, M. G. Bowden, S. A. Kautz, and R. R. Neptune, "Paretic propulsion as a measure of walking performance and functional motor recovery post-stroke: a review," Gait \& posture, vol. 68, pp. 6-14, 2019.

[66] M. D. Lewek, C. Raiti, and A. Doty, "The presence of a paretic propulsion reserve during gait in individuals following stroke," Neurorehabilitation and neural repair, vol. 32, no. 12, pp. 1011-1019, 2018.

[67] K. P. Sannbia, A. L. Ellis, and M.-S. Ko, "Gait deviation associated with absent terminal stance phase in people with stroke," Journal of Korean Society for Neurotherapy, vol. 24, no. 1, pp. 1-9, 2020.

[68] N. Zahradka, K. Verma, A. Behboodi, B. Bodt, H. Wright, and S. C. Lee, "An evaluation of three kinematic methods for gait event detection compared to the kinetic-based 'gold standard',' Sensors, vol. 20, no. 18, p. $5272,2020$. 